\section{New \\ Method}

\title{
Efficacy of the Stonehenge Technique for Minimally Invasive Aortic Valve Replacement via Right Infraaxillary Thoracotomy
}

\author{
Masataka Yamazaki, MD, Hajime Kin, MD, Shohei Kitamoto, MD, Shota Yamanaka, MD, \\ Hidefumi Nishida, MD, Kosaku Nishigawa, MD, and Shuichiro Takanashi, MD
}

\begin{abstract}
Minimally invasive cardiac surgeries for aortic valve replacement (AVR) are still a technical challenge for surgeons because these procedures are undertaken through small incisions and deep surgical fields. Although AVR via vertical infraaxillary thoracotomy can be a cosmetically superior option, a disadvantage of this approach is the distance between the thoracotomy incision and the ascending aorta. Therefore, we devised a technique to perform all manipulations using the fingertips without the aid of a knot pusher or long-shafted surgical instruments. This was achieved by particular placement of several retracted sutures to the right chest wall. We named placement of these sutures the "Stonehenge technique." In conclusion, AVR via vertical infraaxillary thoracotomy with our Stonehenge technique can be safely and simply performed with superior cosmetic advantages.
\end{abstract}

Keywords: Stonehenge technique, aortic valve replacement, minimally invasive cardiac surgery, right infraaxillary mini-thoracotomy

To improve the cosmetic results of minimally invasive cardiac surgery (MICS) for aortic valve replacement (AVR), we use a small right infraaxillary incision. A disadvantage of AVR via right infraaxillary thoracotomy is the distance between the thoracotomy incision and the ascending aorta. Therefore, we devised a technique to perform all manipulations using the fingertips without the aid of a knot pusher or long-shafted surgical instruments. This was achieved by particular placement of several retracted sutures to the right chest wall. We named placement of these sutures the "Stonehenge technique" (Figs. 1A and 1B).

Department of Cardiovascular Surgery, Sakakibara Heart Institute, Tokyo, Japan

Received: October 14, 2016; Accepted: December 21, 2016 Corresponding author: Masataka Yamazaki, MD. Department of Cardiovascular Surgery, Sakakibara Heart Institute, 3-16-1 Asahicho, Fuchu, Tokyo 183-0003, Japan Email: m-yamazaki@mva.biglobe.ne.jp (C)2017 The Editorial Committee of Annals of Thoracic and Cardiovascular Surgery. All rights reserved.

\section{Surgical Technique}

We successfully performed AVR through a small right infraaxillary thoracotomy with our Stonehenge technique in 10 patients between July 2015 and August 2016. The patients were six women and four men with a mean age of 69.3 years (range: 56-81 years). Patients with severe aortic calcification, peripheral arterial stenosis, or poor left ventricular function were not included. The preoperative diagnosis was aortic stenosis in nine patients and aortic regurgitation in one patient.

Using general anesthesia with differential lung ventilation, the patients were placed in a $70^{\circ}$ left lateral position with a pillow beneath the left chest. The right upper arm was abducted anteriorly and the elbow flexed to $90^{\circ}$. The forearm of the patients was set in front of the face and held by a padded positioner bar. An 8-cm skin incision along the edge of the pectoralis major muscle was made at the right anterior axillary line (Fig. 1E). After dissecting a space beneath the pectoralis major muscle anteriorly, a thoracotomy incision was made through the third or fourth intercostal space. Cardiopulmonary bypass was established through the right femoral artery and vein or right atrium. Although the right atrial 


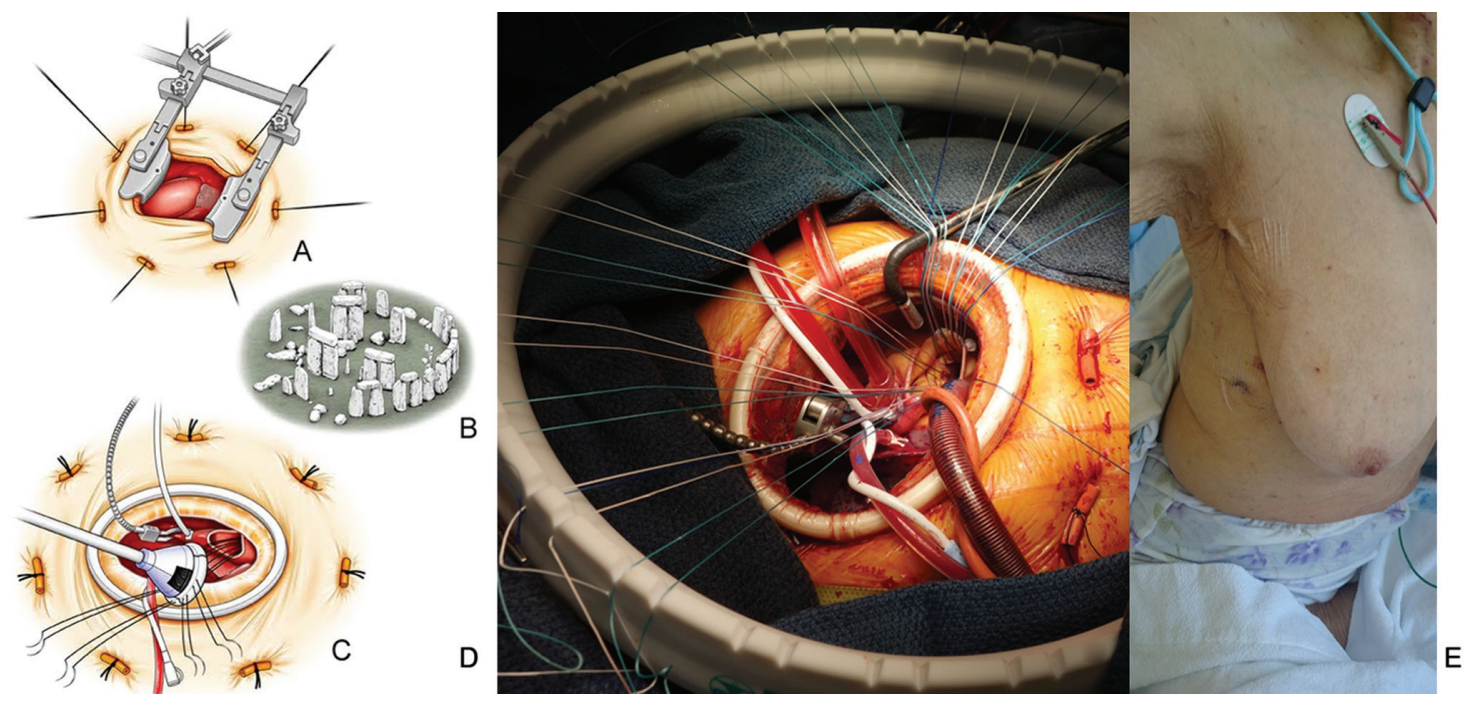

Fig. 1 (A) The pericardium was pulled up by several retracted sutures in a circle formation outside of the wound. These retracted sutures were pulled out of the thorax by an Endo Close needle. (B) We named particular placement of these sutures the Stonehenge technique. (C) Aortic valve replacement via right infraaxillary thoracotomy with our Stonehenge technique can be safely and simply performed. (D) Intraoperative photo of the Stonehenge technique. (E) The right infraaxillary thoracotomy approach had better cosmetic results than traditional approaches through the anterior chest wall.

cannula is better in terms of venous drainage, it will be disturbance in the surgical field. Therefore, we choose the right atrial cannula when cardiopulmonary bypass establishment is difficult through the right femoral vein.

In our series of 10 cases, we experienced no case of poor venous drainage through the right femoral vein or right atrium. Consequently, we do not need the additional superior vena cava (SVC) drainage tube via the right internal jugular vein.

The pericardium was longitudinally opened $2 \mathrm{~cm}$ from the phrenic nerve, and pulled up by several retracted sutures to the right chest wall. All pericardial retracted sutures were pulled out of the thorax by an Endo Close needle (Covidien Inc., Minneapolis, MN, USA) (Figs. 1A and $1 \mathrm{C}$ ). We lifted the ascending aorta and aortic root by these retracted sutures in a circle formation outside of the wound. Therefore, based on the well-known British archaeological site of Stonehenge, we named particular placement of these sutures the Stonehenge technique. A soft tissue protector of size $\mathrm{S}$ was then set. A small rib spreader is optional. A left ventricular venting tube was inserted through a purse string suture placed on the right upper pulmonary vein. An aortic root cannula was placed, and subsequently, the ascending aorta was cross clamped with a Cosgrove flexible shaft clamp inserted through the main incision. Antegrade cold blood cardioplegia was administered through the aortic root, followed by selective administration into each coronary ostium. Retrograde cardioplegia was used as necessary. Oblique aortotomy was performed slightly more distally than usual, and the aortic valve was exposed with stay sutures. The calcified valve was excised from the annulus using long scissors and an ultrasonic aspirator. The prosthetic valve was fixed in place using interrupted 2-0 braided polyester sutures. We could tie down all sutures, including the annular sutures that fix the prosthetic aortic valve, using the fingertips without the aid of a knot pusher. The aortotomy was closed in double layers using 4-0 polypropylene sutures. All of the procedures were performed under direct vision. After weaning the patients off bypass, the pericardium was loosely closed and the chest was closed in the usual manner.

The mean aortic-clamp time was 95 min (range: 76-146 $\mathrm{min}$ ) and mean cardiopulmonary bypass time was $138 \mathrm{~min}$ (range: 117-198 $\mathrm{min}$ ). There were no in-hospital deaths. There were no cases of stroke, perivalvular leak, prolonged ventilation, or excessive bleeding.

\section{Discussion}

MICS for AVR has been developed via partial sternotomy, the parasternal approach, and anterior minithoracotomy by progress of cardiovascular surgery. However, traditional minimally invasive approaches for AVR using anterior chest skin incisions have not necessarily 


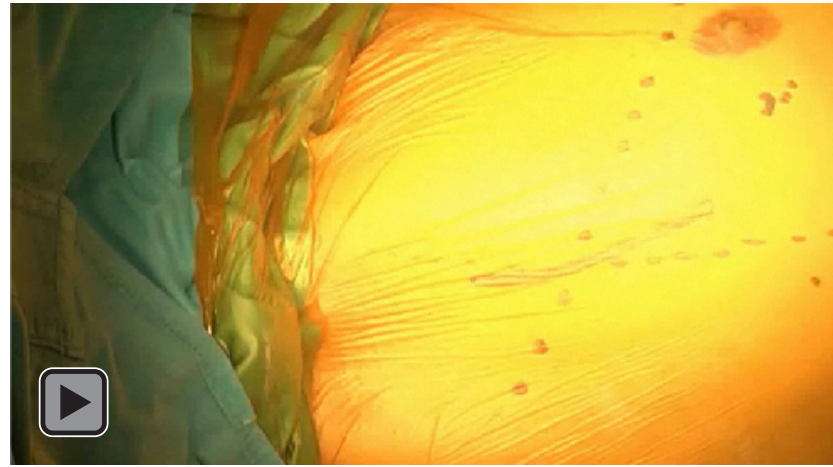

Video 1 Intraoperative video of aortic valve replacement via vertical infraaxillary thoracotomy with our Stonehenge technique (this movie is available online).

shown cosmetic superiority over standard median sternotomy because anterior chest wounds can easily be recognized and tend to lead to hypertrophic scarring. Therefore, MICS for AVR is still not a common procedure compared with MICS for mitral valve surgery.

Ito and associates ${ }^{1,2)}$ used a vertical infraaxillary thoracotomy for aortic valve replacement (TAX-AVR) and limited the small skin incision with endoscopic assistance. Although this TAX-AVR could be a cosmetically superior option for selected patients undergoing AVR, a disadvantage is the distance between the thoracotomy incision and the ascending aorta. Because we cannot manipulate the ascending aorta and aortic valve manually, long-shafted surgical instruments, knot pushers, and videoscopic assistance are mandatory. Notably, TAX-AVR has the potential concern of difficulty in recovering from lethal complications during the operation. As a result, this approach also has not been popular for MICS-AVR.

However, after our experiences with TAX-AVR, we devised a technique to perform all manipulations using the fingertips without the aid of a knot pusher or long-shafted surgical instruments, including a technique of tying down the annular sutures that fix the prosthetic aortic valve (Fig. 1D and Video 1). This was achieved by particular placement of several pericardial retracted sutures in a circle formation outside of the wound (Figs. 1A and 1C). As a result, the ascending aorta and aortic root are lifted to the right chest wall (Figs. 2A and 2B). Special longshafted surgical instruments are no longer necessary because the ascending aorta is drawn close to the wound by this technique.

Our Stonehenge technique has the potential concern of bleeding due to injury of the right internal thoracic artery or the intercostal arteries after cardiopulmonary bypass commenced. However, we could lift the ascending

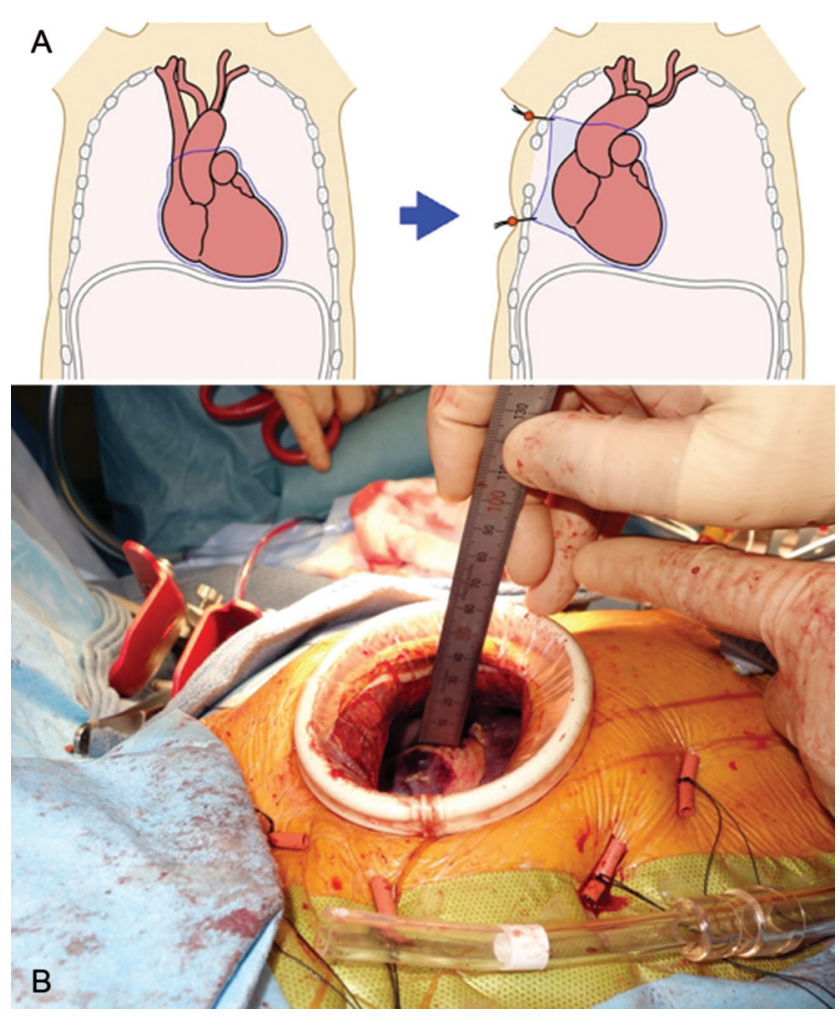

Fig. 2 (A) and (B) Using the Stonehenge technique, the ascending aorta was lifted to $4 \mathrm{~cm}$ from the right chest wall.

aorta and aortic root more strongly using our Stonehenge technique when we decreased patient's cardiac volume by cardiopulmonary bypass.

Moreover, we have no experience of massive bleeding due to Stonehenge technique. As a skin incision along the edge of the pectoralis major muscle was made at the right anterior axillary line, the distance between skin incision and the right internal thoracic artery is far enough.

On the other hand, the insertion of Endo Close needle at the supracostal site is very important to prevent the intercostal artery injury. The biggest complication of this Stonehenge technique is the injury of the right subclavian artery and vein.

The indication of TAX-AVR with our Stonehenge technique is isolated AVR due to aortic valve stenosis or regurgitation. But the case of poor left ventricular function (Ejection fraction $<50 \%$ ) was not included so far. In addition, the risk case of retrograde perfusion through the femoral artery, the severe adhesion of the right thoracic cavity, ascending aorta enlargement (diameter $>40 \mathrm{~mm}$ ), deformity of thoracic cage, and significant shift of aortic root to the left side are contraindication.

We believe that TAX-AVR with our Stonehenge technique could become the standard MICS procedure for 
AVR. In conclusion, TAX-AVR with our Stonehenge technique can be safely and simply performed with superior cosmetic advantages.

\section{Disclosure Statement}

The authors acknowledge no conflict of interest.

\section{References}

1) Ito T, Maekawa A, Hoshino S, et al. Right infraaxillary thoracotomy for minimally invasive aortic valve replacement. Ann Thorac Surg 2013; 96: 715-7.

2) Ito $T$, Maekawa $A$, Hoshino $S$, et al. Right infraaxillary mini-thoracotomy for aortic valve replacement. Ann Cardiothorac Surg 2015; 4: 78-80. 